\title{
HI and $\mathrm{H} \alpha$ Mapping of M31 \& M33
}

\section{Z. S. Kam ${ }^{1}$, C. Carignan ${ }^{1,3,4}$, L. Chemin ${ }^{2}$, O. Hernandez ${ }^{1}$, M. de Denus-Baillargeon ${ }^{1}$ and Y. Djabo ${ }^{1}$}

\author{
${ }^{1}$ Laboratoire d'Astrophysique Expérimentale (LAE), Département de physique, \\ Université de Montréal, C.P. 6128, Succ. Centre-ville, Montréal, Qc, Canada H3C 3J7 \\ ${ }^{2}$ Laboratoire d'Astrophysique de Bordeaux 2, rue de l'Observatoire, \\ BP 89, 33271 Floirac Cedex France. \\ ${ }^{3}$ Observatoire d'Astrophysique de l'Université de Ouagadougou (ODAUO), \\ UFR/SEA, B.P. 7021, Ouagadougou 03, Burkina Faso \\ ${ }^{4}$ Department of Astronomy, University of Cape Town, \\ Private Bag X3, Rondebosh 7701, South Africa \\ email: kam@ASTRO. UMontreal.CA
}

\begin{abstract}
Résumé. We performed a deep Hi and $\mathrm{H} \alpha$ mapping of M31 and M33 in order to get accurate kinematical data of those two galaxies and also to make a comparison between the $\mathrm{HI}$ and $\mathrm{H} \alpha$ kinematics. The HI data were obtained with the DRAO interferometer and the $\mathrm{H} \alpha$ data with the Fabry-Perot system of the Observatoire du mont Mégantic using an EMCCD as a detector. These data will give us the best possible datasets to derive accurate rotation curves and mass models for those two Local Group spirals and provide some new data for the Hil regions studies of these galaxies. While the Hi observations are of low resolution $(\sim 1 \operatorname{arcmin})$, the high resolution of the $\mathrm{H} \alpha$ data $(\sim 1$ arcsec) should allow us to get much more details in the central regions, allowing at the same time a much better determination of the kinematical parameters. Hence, the inner part of the rotation curve, so inportant to constraint properly the mass models, will be determined more accurately.
\end{abstract}

Keywords. Galaxy : individual : M31, M33, Galaxy : kinematics, ISM : lines : Hi, Ho

\section{Introduction and background}

Messier 33 and Messier 31 are very interesting Local Group galaxies. There are, with the Milky Way, the three massive galaxies of the group. These galaxies give us the oportunity to study in great detail the interstellar medium, star formation, galaxy evolution and kinematic and dynamic of galaxies similar to the Milky Way (Klypin et al. 2002). We have thus two real laboratories for testing our models and theories on spiral structure and galaxy formation.

Andromeda was the subject of a large number of studies since 1612 (Brandt 1965; Braun 1991; Braun et al. 2009; Thilker et al. 2005; Carignan et al. 2006; Chemin et al. 2009; Corbelli et al. 2010). The neutral gas in this galaxy is observed with a great interest (Corbelli et al. 2010) and with technics more and more powerful. Interestingly, observations of the Large Magellanic Cloud, despite being a satellite of our Galaxy, may give some clues on the past history of M31 (Kallivayalil et al. 2009; Yang \& Hammer 2010). Hammer et al. (2010) and Yang \& Hammer (2010) have proposed a dynamic model with the MW, M31 and the LMC, which reproduces M31's parameters with the LMC being a tidal dwarf ejected by M31 past merger process. Moreover, a large number of studies (mainly in $\mathrm{HI}$ ) helped to have more information about its spiral arms, the interaction 

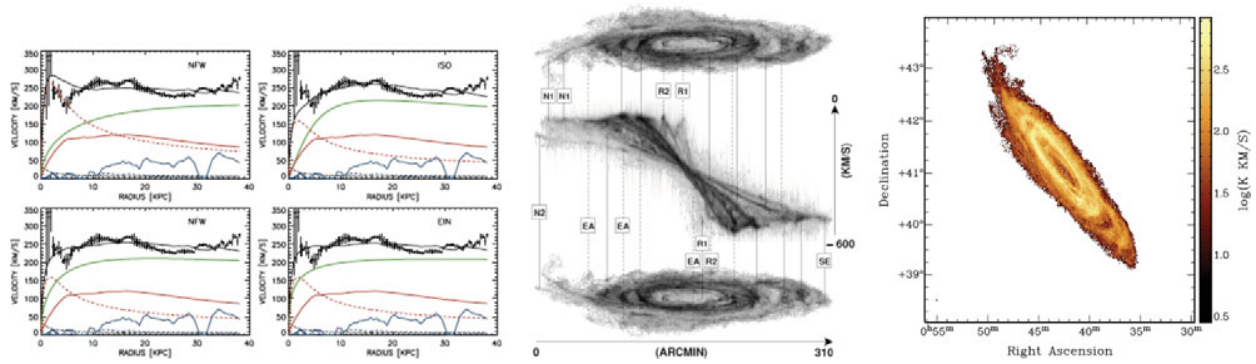

Fig. 1. Hi kinematical results of M31 (Chemin et al. 2009) : (left) mass distribution models, (middle) 3D data cube showing its structures, (right) Hi distribution.

with M32, its warped disk or its ring-like structures. Despite the low HI resolution in the inner part of M31, it is still possible to get some information about its the dark matter content (Chemin et al. 2009).

The Triangulum Galaxy (M33) is the third most massive galaxy of the Local Group. With a mass $\sim 10$ times lower than M31 and the MW, M33 is not as well studied as its two neighbours. The major studies on M33 related to the molecular gas (CO), neutral and ionised gas, in some well known HII regions (Boulesteix et al. 1974; Lagrois \& Joncas 2009). As is the case for M31, there is a lack of $\mathrm{H} \alpha$ kinematical studies at high resolution in the inner part of M33.

In this paper, we first discussed the Hi kinematical studies of our Galaxy neighbours (Carignan et al. 2006; Chemin et al. 2009) and, in the next two sections, the Hi and $\mathrm{H} \alpha$ observations are presented in more detail. The Hi observations were taken with the DRAO radio interferometer and the $\mathrm{H} \alpha$ with the $1.6 \mathrm{~m}$ telescope of the Observatoire du Mont Mégantic, using an optical Fabry-Perot system.

\section{HI Kinematics of M31 and M33}

The Hi observations allow to get the kinematics on large scales. We already have those sensitive Hi observations from DRAO observations : Chemin et al. (2009) for M31; Chemin (in preparation) for M33.

\subsection{M31}

The Hi observations of M31 were the motor of this study. In fact, the Hi velocities in M31 show peculiarities both at small and large radii (Chemin et al. 2009). Using those DRAO observations, Chemin et al. (2009) showed some new structures and also an increasing rotation curve at large radius. Part of his work is represented in figure 1 . This figure shows on the left some velocities curves, with increasing velocities at large radii, and decreasing velocities at the center as well as at $\sim 30 \mathrm{kpc}$. These curves present also some difficulties to represent the inner part of the galaxy even using different mass models. Moreover, the Hi profiles cannot explain the high velocities or components revealed by the $\mathrm{CO}$ observations. So another question is : what will we find for the $\mathrm{H} \alpha$ velocities at small radius? Will they agree with the $\mathrm{CO}$ observations and $\mathrm{CO}$ velocities or with the Hı ? 


\section{2. М33}

Messier 33 has been observed in 2008 and 2009 with DRAO at 1 arcmin resolution and with a spectral sampling of $1 \mathrm{~km} . \mathrm{s}^{-1}$. Six DRAO fields were used to map the whole galaxy, with about 200 hours of integration per field. Single dish Arecibo Hi observations from Putman et al. (2009) have also been used to recover shorter spacings. The first image of Figure 2 shows the preliminary integrated Hi emission of M33. The bright inner disk of M33 is clearly identified as well as diffuse Hi streams located in the north-western and south-eastern outer regions of the disk. Those streams correspond to the HI warp of the galaxy. Hi column densities down to about $5 \times 10^{19}$ atoms.cm ${ }^{-2}$ are observed in the warped structure (Chemin et al. in preparation).

\section{H $\alpha$ mapping and kinematics of M31 and M33}

$\mathrm{H} \alpha$ observations have most of the time better resolution in the inner part of the galaxies. The observations in the $\mathrm{H} \alpha$ band were obtained at the OMM in the summer 2010 and more observations are to come in the 2011 summer. The OMM has a $1.6 \mathrm{~m}$ RitcheyChrétien telescope, located at the summit of mont Mégantic at an altitude of $1111 \mathrm{~m}$. We started observing M33 with an EMCCD, using the CCD Controller for Counting Photons (CCCP) (Daigle et al. 2008, 2009). With CCCP, it is possible to reduce the Clock Induced Charge (CIC - the major source of noise when the EMCCD is operated at high gain). This capability allows for greater efficiency in photon counting mode. Its low CIC rate renders possible the detection of faint fluxes down to $\sim 0.001$ photon/pixel/image (Daigle et al. 2009). The camera has a field of $\sim 9.1^{\prime}$ (the pixel scale $\sim 1.0 \mathrm{arcsec} / \mathrm{pix}$ ). Each field was exposed for one hour. Frames were taken with 0.5 seconde/image, 10 images/channel and 48 chanels/cycle. The interference filter had a central wavelength of $\lambda_{c}=6565.0 \AA$, and a FWHM of $15 \AA$. Maximum transmission was $\sim 80 \%$. The FP interference order was $\mathrm{p}=765$ at $\mathrm{H} \alpha$, with a Free Spectral Range (FSR) of $8.58 \AA(391.77$ $\mathrm{km} \mathrm{s}^{1}$ ). The mean Finesse during the observations was $\sim 16.5$, with an etalon spectral resolution of $\sim 0.53 \AA(\Delta \lambda=\operatorname{FSR}(\AA) /$ Finesse $)$.

We expose also 20 to 30 minutes on the sky in order to subtract efficiently sky lines during the reduction. Between the observations of each field, a calibration cube was obtained. For the M33 mapping with this camera, we have defined more than thirty fields to allow overlap for mosaicing. The reduction system consists of the creation of a wavelength-sorted data cube corrected for airmass dependance, guiding shift, cosmic rays, dark, noise and gain. It takes also into account, ghosts correction (see Epinat 2008), heliocentric velocity correction, hanning spectral smoothing and sky subtraction (Daigle et al. 2006). Options as spatial binning procedure, single gaussian fitting procedure, deconvolution of the profile for the filter dependance, extraction of the velocity, dispersion and $\mathrm{H} \alpha$ integrated maps are possible (Hlavacek-Larrondo et al. 2010; Daigle et al. 2006). The right image of figure 2 shows, the radial velocity image of the field identified in the middle frame (no smoothing was applied). We use Adhocw to compute the RV maps. We present here one field of the first mosaic of this survey taken with the EMCCD and Fabry-Perot at OMM. These preliminary pictures are not accurate analyses of our survey but they give velocities higher than $120 \pm 10 \mathrm{~km} \cdot \mathrm{s}^{-1}$ in an external field of the galaxy. More accurate values are coming soon (Kam et al.in preparation). 

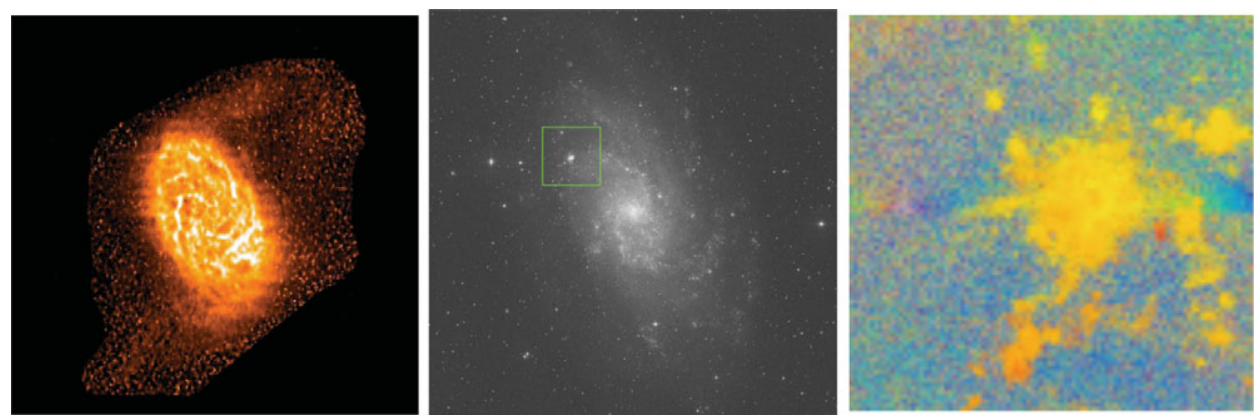

Fig. 2. (left) : preliminary integrated Hi emission of M33 (Chemin et al.in preparation). (central) : DSS bleu image of M33 \& the field of view of the camera is shown by a green square. (right) : preliminary results about the $\mathrm{H} \alpha$ mapping of M33 (Kam et al. in preparation).

\section{Conclusion}

M31 and M33 are two galaxies near the MW,. These galaxies are fairly typical of spiral galaxies. Their kinematics and dynamics give a good indication of other external galaxies, but also a good idea on our own Galaxy. Several studies of the kinematics of M33 and M31 were carried out in $\mathrm{HI}$ and $\mathrm{H} \alpha$. Chemin et al. (2009) and Corbelli et al. (2010), present the most accurate kinematical parameters on large scale using the HI for M31. The results of these studies show the presence of new structures and increasing velocities in the outer parts of the galaxy.

Kinematics from Hi for M33 on is presented by Warner et al. (1973); Huchtmeier (1973), and Westmeier et al. (2005). The Hi does not give us as precise details as $\mathrm{H} \alpha$ because of the low spatial resolution and of beam smearing. Only a few studies at H $\alpha$ exist on those galaxies : (Rubin \& Ford 1970; Boulesteix \& Monnet 1970; Boulesteix et al. 1974 ) presented some studies on those galaxies on small scales. This new mapping at $\mathrm{H} \alpha$ will allow us to calculate more precisely the kinematical parameters for those galaxies and to make a comparison of these results with those at Hi already well-known. In fact, the capacities of our instrument allow to get more details and resolution in galaxies observations (Daigle et al. 2008, 2009).

\section{References}

Boulesteix, J., Courtes, G., Laval, A., Monnet, G., \& Petit, H. 1974, A\&̛A, 37, 33

Boulesteix, J. \& Monnet, G. 1970, A\&A, 9, 350

Brandt, F. 1965, MNRAS, 129, 309

Braun, R. 1991, ApJ, 372, 54

Braun, R., Thilker, D. A., Walterbos, R. A. M., \& Corbelli, E. 2009, ApJ, 695, 937

Carignan, C., Chemin, L., Huchtmeier, W. K., \& Lockman, F. J. 2006, ApJL, 641, L109

Chemin, L., Carignan, C., \& Tyler, F. 2009, ApJ, 705, 1395

Corbelli, E., Lorenzoni, S., Walterbos, R., Braun, R., \& Thilker, D. 2010, A\&A A, 511, A89

Daigle, O., Carignan, C., Gach, J., Guillaume, C., Lessard, S., Fortin, C., \& Blais-Ouellette, S. 2009, PASP, 121, 866

Daigle, O., Carignan, C., Hernandez, O., Chemin, L., \& Amram, P. 2006, MNRAS, 368, 1016

Daigle, O., Gach, J., Guillaume, C., Lessard, S., Carignan, C., \& Blais-Ouellette, S. 2008, in SPIE Conference Series, Vol. 7014, 70146L

Epinat, B. e. a. 2008, MNRAS, 388, 500

Hammer, F., Yang, Y. B., Wang, J., Puech, M., Flores, H., \& Fouquet, S. 2010, ApJ, 725, 542

Hlavacek-Larrondo, J., Carignan, C., Daigle, O., de Denus-Baillargeon, M., Marcelin, M., Epinat, B., \& Hernandez, O. 2010, MNRAS, 1649 
Huchtmeier, W. 1973, A\& A, 22, 91

Kallivayalil, N., Besla, G., Sanderson, R., \& Alcock, C. 2009, ApJ, 700, 924

Klypin, A., Zhao, H., \& Somerville, R. S. 2002, ApJ, 573, 597

Lagrois, D. \& Joncas, G. 2009, ApJ, 700, 1847

Putman, M. E., Peek, J. E. G., Muratov, A., Gnedin, O. Y., Hsu, W., Douglas, K. A., Heiles, C., Stanimirovic, S., Korpela, E. J., \& Gibson, S. J. 2009, ApJ, 703, 1486

Rubin, V. C. \& Ford, Jr., W. K. 1970, ApJ, 159, 379

Thilker, D. A., Braun, R., \& Westmeier, T. 2005, in ASP Conference Series, Vol. 331, ExtraPlanar Gas, ed. R. Braun, 113

Warner, P. J., Wright, M. C. H., \& Baldwin, J. E. 1973, MNRAS, 163, 163

Westmeier, T., Braun, R., \& Thilker, D. 2005, Astronomy and Astrophysics, 436, 15

Yang, Y. \& Hammer, F. 2010, ApJL, 725, L24 